\title{
Preferencias de los estudiantes de medicina de la Universidad de Buenos Aires en la elección de la especialidad
}

\author{
Raúl A. Borracci, Ricardo L. Poveda-Camargo, Roberto D. Pittaluga, Eduardo B. Arribalzaga, Pedro Ferraina
}

Introducción. Los factores que influyen en la elección de una especialidad son la demanda laboral, la remuneración, el prestigio, el tiempo de formación y la posibilidad de tener un estilo de vida 'controlable'. Sin embargo, la influencia de la cultura podría modificar el peso de estas variables a la hora de elegir una especialidad.

Objetivo. Analizar los factores que influían sobre la elección de la especialidad médica por parte de los estudiantes.

Sujetos y métodos. Entre marzo y mayo de 2010 se encuestaron 125 estudiantes que cursaban quinto año o el internado. Por medio de una encuesta anónima se recogió información sobre los factores que podrían influir en la elección futura de la especialidad.

Resultados. La pediatría fue la especialidad más elegida, pero se la consideró entre las menos lucrativas, con más tiempo de formación y prestigio moderado, aunque con una adecuada demanda laboral. La cirugía fue la segunda más elegida y considerada como la de mayor prestigio y mejor remunerada. La clínica médica requeriría más tiempo de formación, con más oportunidades laborales, pero peor remuneradas. Tanto la clínica médica como la cirugía se eligieron como las de estilo de vida menos 'controlable', hallándose en tercer lugar la pediatría.

Conclusión. Las características de remuneración, prestigio, estilo de vida, tiempo de formación y demanda laboral, en general, parecen no haber influido significativamente en la elección de la especialidad.

Palabras clave. Elección de la especialidad. Estudiantes. Medicina. Pregrado. Profesión.

\section{Medical students' preferences on specialty selection in Buenos Aires University}

Introduction. Factors associated with medical students' specialty choice are work market opportunities, expected income, prestige, length of training and controllable life style possibility. However, cultural influence may modify the weight of these variables at time of choosing a specialty.

Aim. To analyze the factors influencing on medical students' specialty preferences.

Subjects and methods. From March to May of 2010, 125 medical students' from 5th and 6th year were surveyed. Information was collected by an anonymous questionnaire about factors influencing on a future specialty choice.

Results. Opinions about which specialties were regarded as better remunerate, has most prestige, a controllable life style, need more length of training and/or has a good job market were obtained. Pediatrics was the most selected but it was considered one with the worst payment, with less length of training, moderate prestige, although an adequate job market. Surgery was the second choice and was regarded as the one with most prestige and best remuneration. On the other hand, internal medicine was considered to need more length of training, with better work opportunities, but worse remuneration. Internal medicine as well as surgery, is regarded as having the less controllable life style, being pediatrics on third place.

Conclusion. The characteristics of income, prestige, life style, length of training and job market opportunities, in general seem to not significantly relate on specialty selection.

Key words. Medical students. Medicine. Pregraduate. Profession. Specialty choice.

\section{Introducción}

Entre las variables que más parecen influir en la elección de una carrera o especialidad se hallan la de- manda del mercado, la remuneración percibida, el prestigio de la profesión, el tiempo requerido para la formación y la capacidad de generar un estilo de vida 'controlable' que minimice las urgencias y la
Hospital de Clínicas. Facultad de Medicina. Universidad de Buenos Aires. Buenos Aires, Argentina.

Correspondencia: Dr. Raúl A. Borracci. La Pampa, 3030, 1. ${ }^{\circ}$ B. 1428 Buenos Aires, Argentina.

E-mail:

raborracci@gmail.com

Conflicto de intereses: No declarado.

Conflict of interests: None declared.

(C) 2012 Educación Médica 
Tabla I. Número de alumnos identificados por año de curso y sexo.

\begin{tabular}{lccc}
\hline & Varones & Mujeres & Totales \\
\hline $5^{\circ}$ año & $11(26,2 \%)$ & $31(73,8 \%)$ & 42 \\
\hline $6^{\circ}$ año (internado) & $21(32,8 \%)$ & $43(67,2 \%)$ & 64 \\
\hline Totales & $32(30,2 \%)$ & $74(69,8 \%)$ & 106 \\
\hline
\end{tabular}

actividad laboral no programada [1-4]. En estudios previos se ha observado que, en mayor medida los varones que las mujeres, se tiende a elegir especialidades con el estilo de vida 'controlable' [2]. En el contexto de las especialidades médicas, se define un estilo de vida 'controlable' con las siguientes características: tiempo libre para el descanso, la vida familiar y las actividades vocacionales, y control de las horas semanales dedicadas a las responsabilidades profesionales [3]. Según algunos autores, estos aspectos del estilo de vida podrían tener más influencia en la elección de la especialidad que la remuneración, el prestigio o el tiempo de formación y entrenamiento requerido [5-8]. De todas formas, otros estudios sugieren que no sólo el estilo de vida sino también los ingresos económicos influyen en la elección de la carrera y la especialidad. Newton et al [9] refieren que los estudiantes identificaron la ginecología y obstetricia como la especialidad menos 'amigable' en términos de estilo de vida e ingresos, y en el otro extremo, la radiología (diagnóstico por imagen) como la más 'amigable'.

Aunque estos factores han sido estudiados y resaltados por varios investigadores, la influencia de la cultura podría modificar el peso de estas variables a la hora de elegir una especialidad. El trabajo de Avgerinos et al [10], por ejemplo, demostró que la demanda, la remuneración y el prestigio fueron los factores que menos influyeron en un grupo de estudiantes griegos. Por otra parte, en un trabajo previo analizamos las expectativas de los estudiantes de medicina de la Universidad de Buenos Aires sobre su práctica profesional futura de acuerdo con la especialidad elegida [11]. En este estudio hallamos que las especialidades más elegidas parecían depender del proceso de feminización de la matrícula médica: en las primeras cuatro posiciones se situaban pediatría, clínica, ginecología y obstetricia, y psiquiatría. Así mismo, los encuestados manifestaron unas expectativas económicas, a corto y medio plazo, mayores que la verdadera renta actual de los médicos en Argentina, y a la vez destacaba la intención de participar activamente en la docencia, aunque se mostraba escaso interés por la investigación. En nuestra población de estudiantes no ha quedado claro cuáles son las principales motivaciones o influencias para optar por una especialidad, habida cuenta de que entre las más elegidas se encuentran especialidades con una sobredimensión de profesionales, remuneraciones bajas y estilos de vida menos 'controlables'. En este nuevo trabajo, el objetivo fue analizar cuáles eran los factores que influían sobre la elección de la especialidad médica por parte de los estudiantes.

\section{Sujetos y métodos}

Entre marzo y mayo de 2010 se encuestaron 125 estudiantes de medicina de la Universidad de Buenos Aires que cursaban el quinto año de la carrera o el internado anual rotatorio previo a la graduación. La distribución por año y sexo se muestra en la tabla I. Por medio de una encuesta anónima se recogió información sobre los factores que podrían influir en la elección futura de la especialidad. Para ello se obtuvieron las opiniones de los encuestados con respecto a qué especialidades consideraban que estaban mejor remuneradas, gozaban de mayor prestigio social, tenían un estilo de vida más 'controlable', requerían más tiempo de formación o tenían más demanda de trabajo en el mercado laboral. Desde el punto de vista operativo, y a fin de facilitar las respuestas, el estilo de vida 'controlable' se definió como la condición de tener menos urgencias, actividad nocturna o guardias. Simultáneamente se recogieron datos sobre la edad, el sexo, la especialidad a elegir y el tiempo esperable de dedicación a la profesión y a las actividades familiares y recreativas.

Las variables se expresaron como valores absolutos, porcentajes, medias y desviaciones estándares según correspondiera al tipo de datos. La comparación de los datos continuos se realizó con $t$ de Student, y los cualitativos, con $\chi^{2}$ o probabilidad exacta de Fisher $(p)$ para dos colas. Para analizar cuáles eran las condiciones y creencias de los estudiantes que influían sobre la elección de la especialidad, se compararon las opiniones que tenían sobre ella entre aquellos que la habían elegido y aquellos que no. Se asumió que si las opiniones no diferían entre los grupos, no existiría una influencia particular de ese factor para determinar la elección de dicha especialidad. Para el análisis se usó el programa estadístico SPSS v. 10.0 y la hoja de cálculo Microsoft Office Excel 2003. 
Tabla II. Especialidades elegidas por los encuestados $(n=106)$.

\begin{tabular}{|c|c|c|c|c|c|c|c|}
\hline \multirow[b]{2}{*}{ No sabe aún } & \multicolumn{2}{|c|}{$\begin{array}{c}\text { Total } \\
(n=106)\end{array}$} & \multicolumn{2}{|c|}{$\begin{array}{l}5 .^{\circ} \text { año } \\
(n=42)\end{array}$} & \multicolumn{2}{|c|}{$\begin{array}{l}6 .{ }^{\circ} \text { año } \\
(n=64)\end{array}$} & \multirow{2}{*}{$\begin{array}{c}p^{\mathrm{a}} \\
0,042\end{array}$} \\
\hline & 16 & $15,1 \%$ & 10 & $23,8 \%$ & 6 & $9,4 \%$ & \\
\hline Pediatría & 16 & $15,1 \%$ & 1 & $2,3 \%$ & 15 & $23,4 \%$ & 0,007 \\
\hline Cirugía & 9 & $8,5 \%$ & 4 & $9,5 \%$ & 5 & $7,8 \%$ & 0,737 \\
\hline Clínica médica & 8 & $7,5 \%$ & 7 & $16,7 \%$ & 1 & $1,6 \%$ & 0,006 \\
\hline Ginecología-obstetricia & 8 & $7,5 \%$ & 1 & $2,3 \%$ & 7 & $10,9 \%$ & 0,142 \\
\hline Cardiología & 7 & $6,6 \%$ & 2 & $4,8 \%$ & 5 & $7,8 \%$ & 0,701 \\
\hline Psiquiatría & 6 & $5,7 \%$ & 2 & $4,8 \%$ & 4 & $6,3 \%$ & 1,000 \\
\hline Traumatología & 6 & $5,7 \%$ & 1 & $2,3 \%$ & 5 & $7,8 \%$ & 0,399 \\
\hline Dermatología & 6 & $5,7 \%$ & 4 & $9,5 \%$ & 2 & $3,1 \%$ & 0,211 \\
\hline Infectología & 4 & $3,8 \%$ & 3 & $7,1 \%$ & 1 & $1,6 \%$ & 0,298 \\
\hline Oftalmología & 3 & $2,8 \%$ & 0 & $0,0 \%$ & 3 & $4,7 \%$ & 0,275 \\
\hline Anestesiología & 2 & $1,9 \%$ & 2 & $4,8 \%$ & 0 & $0,0 \%$ & 0,155 \\
\hline Diagnóstico por imagen & 2 & $1,9 \%$ & 0 & $0,0 \%$ & 2 & $3,1 \%$ & 0,517 \\
\hline Neurocirugía & 2 & $1,9 \%$ & 0 & $0,0 \%$ & 2 & $3,1 \%$ & 0,517 \\
\hline Neurología & 2 & $1,9 \%$ & 2 & $4,8 \%$ & 0 & $0,0 \%$ & 0,155 \\
\hline Otorrinolaringología & 2 & $1,9 \%$ & 0 & $0,0 \%$ & 2 & $3,1 \%$ & 0,517 \\
\hline Oncología & 1 & $0,9 \%$ & 1 & $2,3 \%$ & 0 & $0,0 \%$ & 0,396 \\
\hline Otras especialidades & 6 & $5,7 \%$ & 2 & $4,8 \%$ & 4 & $6,3 \%$ & 1,000 \\
\hline
\end{tabular}

\section{Resultados}

Respondieron la encuesta 106 alumnos (84,8\%), de los cuales el $60,4 \%(n=64)$ pertenecía a sexto año (internado rotatorio), y el resto, a quinto año. La edad media era de 25,90 \pm 2,28 años (rango: 23-39 años) y la distribución por sexo era del $69,8 \%(n=74)$ de mujeres y el 30,2\% $(n=32)$ de varones.

Sólo en pediatría se observó una relación estadísticamente significativa entre la elección de dicha especialidad y el sexo, ya que fue elegida con más frecuencia por mujeres que por varones: $18,9 \%(14 / 74)$ frente a $3,1 \%(1 / 32)$, respectivamente $(p=0,035)$.
Aunque los valores no alcanzaron significación estadística, una tendencia similar, a favor de los varones en este caso, se observó en cirugía: 15,6\% (5/32) de varones frente a $5,4 \%(4 / 74)$ de mujeres $(p=0,125)$.

En la tabla II se enumeran las especialidades elegidas y en la tabla III se resumen las opiniones sobre las especialidades que aparecían en la encuesta, que permitía escoger simultáneamente entre varias posibilidades. Otras opiniones mostraron que pediatría era la peor remunerada. Oftalmología, otorrinolaringología y diagnóstico por imagen se consideraron las de menor prestigio, mientras que el estilo de vida más 'controlable' favoreció nuevamente a of- 
Tabla III. Resumen de las opiniones sobre las principales especialidades.

\begin{tabular}{|c|c|c|c|c|c|}
\hline & $\%$ & $n$ & & $\%$ & $n$ \\
\hline \multicolumn{3}{|l|}{ Especialidades mejor remuneradas } & \multirow{2}{*}{\multicolumn{3}{|c|}{$\begin{array}{l}\text { Especialidades con estilo } \\
\text { de vida menos controlable }\end{array}$}} \\
\hline Anestesiología & $55,7 \%$ & 59 & & & \\
\hline Cirugía & $45,3 \%$ & 48 & Diagnóstico por imagen & $2,8 \%$ & 3 \\
\hline \multirow{2}{*}{ Oftalmología } & $45,3 \%$ & 48 & Oncología & $0,9 \%$ & 1 \\
\hline & \multirow{2}{*}{$24,4 \%$} & \multirow{2}{*}{29} & Anestesiología & $0,9 \%$ & 1 \\
\hline Diagnóstico por imagen & & & Oftalmología & $0,9 \%$ & 1 \\
\hline Cardiología & $9,4 \%$ & 10 & Dermatología & $0,0 \%$ & 0 \\
\hline Oncología & $9,4 \%$ & 10 & Infectología & $0,0 \%$ & 0 \\
\hline Traumatología & $8,5 \%$ & 9 & Otorrinolaringología & $0,0 \%$ & 0 \\
\hline Otorrinolaringología & $5,7 \%$ & \multirow{2}{*}{5} & \multirow{2}{*}{$\begin{array}{l}\text { Especialidades que requieren } \\
\text { más tiempo de formación }\end{array}$} & & \\
\hline Ginecología-obstetricia & $4,7 \%$ & & & & \\
\hline Psiquiatría & $4,7 \%$ & 5 & Cirugía & $84,9 \%$ & 90 \\
\hline Dermatología & $4,7 \%$ & 5 & Clínica médica & $45,3 \%$ & 48 \\
\hline Clínica médica & $0,9 \%$ & 1 & Pediatría & $38,7 \%$ & 41 \\
\hline Pediatría & $0,0 \%$ & 0 & Cardiología & $21,7 \%$ & 23 \\
\hline Infectología & $0,0 \%$ & 0 & Oncología & $16,0 \%$ & 17 \\
\hline \multicolumn{3}{|l|}{ Especialidades con mayor prestigio } & Diagnóstico por imagen & $13,2 \%$ & 14 \\
\hline Cirugía & $86,8 \%$ & 92 & Ginecología-obstetricia & $10,4 \%$ & 11 \\
\hline Cardiología & $47,2 \%$ & 50 & Psiquiatría & $7,5 \%$ & 8 \\
\hline Pediatría & $27,4 \%$ & 29 & Traumatología & $5,7 \%$ & 6 \\
\hline \multirow[t]{2}{*}{ Clínica médica } & $15,1 \%$ & 16 & Oftalmología & $2,8 \%$ & 3 \\
\hline & $14,2 \%$ & 15 & Infectología & $0,9 \%$ & 1 \\
\hline Traumatología & $4,7 \%$ & 5 & Otorrinolaringología & $0,9 \%$ & 1 \\
\hline Cinecología-obstetricia & $4 \%$ & 5 & Dermatología & $0,0 \%$ & 0 \\
\hline Uiteculugia-unstetilia & & & Anestesiología & $0,0 \%$ & 0 \\
\hline Anestesiología & $0,9 \%$ & 1 & $\begin{array}{l}\text { Especialidades con } \\
\text { más demanda de trabajo }\end{array}$ & & \\
\hline Dermatología & $0,0 \%$ & 0 & Clínica médica & $46,2 \%$ & 49 \\
\hline Infectología & $0,0 \%$ & 0 & Pediatría & $44,3 \%$ & 47 \\
\hline Oftalmología & $0,0 \%$ & 0 & Diagnóstico por imagen & $33,0 \%$ & 35 \\
\hline Otorrinolaringología & $0,0 \%$ & 0 & Cirugía & $25,5 \%$ & 27 \\
\hline Diagnóstico por imagen & $0,0 \%$ & 0 & Cardiología & $20,7 \%$ & 22 \\
\hline Especialidades con estilo & & & Oftalmología & $20,7 \%$ & 22 \\
\hline de vida menos controlable & & & Ginecología-obstetricia & $19,8 \%$ & 21 \\
\hline Clínica médica & $72,6 \%$ & 77 & Oncología & $18,9 \%$ & 20 \\
\hline Cirugía & $60,4 \%$ & 64 & Psiquiatría & $13,2 \%$ & 14 \\
\hline Pediatría & $45,3 \%$ & 48 & Traumatología & $11,3 \%$ & 12 \\
\hline Ginecología-obstetricia & $40,6 \%$ & 43 & Anestesiología & $5,7 \%$ & 6 \\
\hline Traumatología & $39,6 \%$ & 42 & Otorrinolaringología & $4,7 \%$ & 5 \\
\hline Cardiología & $17,9 \%$ & 19 & Dermatología & $2,8 \%$ & 3 \\
\hline Psiquiatría & $2,8 \%$ & 3 & Infectología & $0,0 \%$ & 0 \\
\hline
\end{tabular}


talmología, otorrinolaringología y oncología. Por su parte, los encuestados opinaron que oftalmología y otorrinolaringología requerían menos tiempo de formación, y que dermatología y otorrinolaringología tenían menos demanda de trabajo.

En la tabla IV se analizó la relación entre la especialidad elegida y la opinión que los encuestados tenían sobre ella. En todos los casos, los valores no significativos indicaron que no se pudo encontrar relación entre la elección de la especialidad y los aspectos enumerados en la primera columna, lo que no significa que no exista, habida cuenta de la posibilidad de un error $\beta$ alto dependiente del tamaño de la muestra. Tal vez una muestra de mayor tamaño podría haber detectado alguna relación. A modo de ejemplo, quienes eligieron pediatría no opinaron distinto de quienes no optaron por dicha especialidad, es decir, la condición de mejor remuneración o prestigio, el estilo de vida menos 'controlable' y el mayor tiempo de formación o de demanda de trabajo no se consideraron particularmente para elegir la especialidad. Lo mismo pareció ocurrir con el resto de las especialidades mencionadas en la tabla IV. Como excepción, quienes eligieron clínica médica opinaron que esta especialidad requería más tiempo de formación $(7 / 8)$, en comparación con quienes no optaron por la especialidad (41/98) ( $p=0,022$; coeficiente de contingencia $=0,236 ; V$ de Cramer $=0,242$ ).

Por último, los encuestados refirieron que dedicarían en promedio 50,0 $\pm 13,8$ h semanales (rango: 8-70 h) a la actividad médica y $53,7 \pm 19,1 \mathrm{~h}$ semanales (rango: 10-90 h) al ocio y la familia.

\section{Discusión}

En términos generales, las características de remuneración, prestigio, estilo de vida, tiempo de formación y demanda laboral parecen no haber influido significativamente en la toma de decisión de los estudiantes encuestados para elegir la especialidad. La opinión que tuvo cada encuestado sobre la especialidad elegida por él no fue significativamente diferente de la opinión de los demás (Tabla IV), lo que induce a pensar que ninguna de las características analizadas (remuneración, prestigio, estilo de vida, etc.) tuvo una influencia mayor para decidir la elección de la especialidad. En particular, especialidades como pediatría y clínica médica fueron las más elegidas, a pesar de estar entre las peor remuneradas, con más tiempo requerido para la formación y un estilo de vida menos 'controlable'. En el caso de la cirugía, la remuneración y el prestigio podrían tener alguna influencia en la elección de dicha especiali-
Tabla IV. Valores de $p$ y absolutos obtenidos al relacionar las cuatro especialidades más elegidas y la opinión que los encuestados tenían sobre dichas especialidades. Los valores no significativos indican que no se pudo detectar relación entre la elección de la especialidad y los aspectos enumerados en la primera columna.

\begin{tabular}{|c|c|c|c|c|}
\hline & Pediatría & Cirugía & $\begin{array}{l}\text { Clínica } \\
\text { médica }\end{array}$ & $\begin{array}{c}\text { Ginecología- } \\
\text { obstetricia }\end{array}$ \\
\hline \multirow{2}{*}{ Mejor remunerada } & 1,000 & 0,730 & 1,000 & 1,000 \\
\hline & $0 / 16$ vs $0 / 90$ & $3 / 9$ vs $42 / 97$ & $0 / 8$ vs $1 / 98$ & $0 / 8$ vs $5 / 93$ \\
\hline \multirow{2}{*}{ Mayor prestigio } & 1,000 & 0,603 & 1,000 & 1,000 \\
\hline & $4 / 16$ vs $25 / 90$ & $9 / 9$ vs $83 / 97$ & $1 / 8$ vs $15 / 98$ & $0 / 8$ vs $5 / 93$ \\
\hline \multirow{2}{*}{ Peor estilo de vida } & $0,339^{a}$ & 1,000 & 0,441 & 0,712 \\
\hline & $9 / 16$ vs 39/90 & $6 / 9$ vs 58/97 & $7 / 8$ vs $70 / 98$ & $4 / 8$ vs $39 / 93$ \\
\hline \multirow{2}{*}{$\begin{array}{l}\text { Más tiempo } \\
\text { de formación }\end{array}$} & $0,916^{a}$ & 1,000 & 0,021 & 1,000 \\
\hline & $6 / 16$ vs $35 / 90$ & $8 / 9$ vs $82 / 97$ & $7 / 8$ vs $41 / 98$ & $1 / 8$ vs $10 / 93$ \\
\hline \multirow{2}{*}{$\begin{array}{l}\text { Mayor demanda } \\
\text { de trabajo }\end{array}$} & $0,226^{a}$ & 1,000 & 0,703 & 0,192 \\
\hline & $9 / 16$ vs $36 / 90$ & $2 / 9$ vs $24 / 97$ & $4 / 8$ vs $45 / 98$ & $3 / 8$ vs $18 / 93$ \\
\hline
\end{tabular}

La fila superior de cada ítem corresponde al valor de $p$, mientras que la inferior señala los valores absolutos de las opiniones de quienes eligieron la especialidad frente a (vs) quienes no la eligieron. ${ }^{a} \chi^{2}$, y el resto, $p$ de Fisher para dos colas.

dad. Es importante destacar que la ausencia de relación estadística entre la especialidad elegida y los factores analizados podrían depender de una baja potencia de la muestra para detectar diferencias estadísticas. Este punto ha de considerarse como una limitación del estudio. Por ejemplo, en el caso de las dos relaciones con valores $p$ más cercanos a la significación estadística, la potencia de la muestra fue del $60 \%$ al comparar la relación entre la mayor demanda laboral y la especialidad de ginecología-obstetricia $(p=0,192)$, mientras que la misma potencia alcanzó sólo el $40 \%$ al cotejar también la demanda de trabajo con la especialidad de pediatría $(p=0,226)$.

En contraposición a nuestro hallazgo, las publicaciones de la última década en general tienden a demostrar que las actitudes de los estudiantes en vista a la elección de una especialidad han cambiado a favor de optar por un estilo de vida con una actividad laboral más 'controlable'. Así, Gelfand et al [6] estudiaron el declive del interés por la cirugía por parte de los estudiantes de Estados Unidos. Concluyeron que los estudiantes preferían elegir especialidades con un estilo de vida 'más amigable', o sea, que tuvieran menos estrés, más tiempo libre 
y menos cantidad de actividad y compromiso laboral. Si bien el estilo de vida y los ingresos económicos parecen ser factores determinantes para influir en la elección de la especialidad, otros autores han demostrado que el peso dado a cada factor varía considerablemente según la especialidad de que se trate [9]. Por ejemplo, aunque la cirugía y la ginecología-obstetricia se consideran como especialidades 'no amigables' desde el punto de vista del estilo de vida, quienes optan por ellas lo hacen mayormente por los ingresos más elevados que suelen percibirse. Por su parte, los mismos estudiantes encuestados en ese estudio eligieron el diagnóstico por imagen, oftalmología, anestesiología, urología, dermatología y otorrinolaringología como las especialidades con un estilo de vida más 'controlable', y psiquiatría, pediatría, traumatología y clínica médica, como de estilo de vida intermedio. Estos resultados son consistentes con los hallados en nuestro trabajo, excepto que para nuestros estudiantes, clínica médica, pediatría y traumatología se hallan entre las especialidades con un estilo de vida menos 'controlable. Sin embargo, los resultados presentados por Dorsey et al [4] tras el análisis de los resultados del National Resident Matching Program de Estados Unidos coinciden en gran medida con los de nuestro estudio, ya que también incluyen la clínica médica entre las especialidades con un estilo de vida no 'controlable'.

Como ya se mencionó, y en coincidencia con el trabajo aquí presentado, la influencia de la cultura podría modificar el peso de los distintos factores analizados a la hora de elegir una especialidad. En este sentido, un estudio concluyó que la elección de la especialidad de medicina familiar parece estar influida por el contexto cultural del estudiante. Así, los estudiantes que consideran que la atención primaria en salud es importante, tienen en general menores expectativas económicas y no planean en general una carrera académica o de investigación [1].

En conclusión, esta encuesta reveló que factores de peso en poblaciones de estudiantes de otros países, como la remuneración, el prestigio, el estilo de vida, el tiempo de formación y la demanda laboral, en general parecen no haber influido significativamente en la elección de la especialidad en un grupo local de estudiantes de medicina próximo a graduarse. Si bien estos resultados podrían estar sesgados por la potencia de la muestra empleada, la única relación estadística esperable en un estudio de mayores dimensiones podría asociar la expectativa de una mayor demanda laboral y la elección de ciertas especialidades, como ginecología-obstetricia y pediatría. Otros aspectos sociales, culturales o vocacionales no analizados en este estudio podrían vincularse con las preferencias por una u otra especialidad. La falta de información sobre la verdadera situación de la práctica médica en el país, la ausencia de cupos de ingreso en las especialidades y el proceso de feminización de la matrícula se hallarían entre los factores locales que podrían tener influencia a la hora de elegir una especialidad.

Bibliografía

1. Senf JH, Campos-Outcalt D, Kutob R. Factors related to the choice of family medicine: a reassessment and literature review. J Am Board Fam Pract 2003; 16: 502-12.

2. Lambert EM, Holmboe E. The relationship between specialty choice and gender of U.S. medical students, 1990-2003. Acad Med 2005; 80: 797-802.

3. Hauer KE, Durning SJ, Kernan WN, Fagan MJ, Mintz M, O'Sullivan PS, et al. Factors associated with medical students' career choices regarding internal medicine. JAMA 2008; 300: 1154-64.

4. Dorsey ER, Jarjoura D, Rutecki GW. Influence of controllable lifestyle on recent trends in specialty choice by US medical students. JAMA 2003; 290: 1173-8.

5. Schwartz RW, Haley JV, Williams C, Jarecky RK, Strodel WE, Young B, et al. The controllable lifestyle factor and students' attitudes about specialty selection. Acad Med 1990; 55: 207-10.

6. Gelfand DV, Podnos YD, Wilson SE, Cooke J, Williams RA. Choosing general surgery: insights into career choices of current medical students. Arch Surg 2002; 137: 941-7.

7. Schwartz RW, Jarecky RK, Strodel WE, Haley JV, Young B, Griffen WO. Controllable lifestyle: a new factor in career choice by medical students. Acad Med 1989; 64: 606-9.

8. Jarecky RK, Schwartz RW, Haley JV, Donnelly MB. Stability of medical specialty selection at the University of Kentucky. Acad Med 1991; 66: 756-61.

9. Newton DA, Grayson MS, Thompson LF. The variable influence of lifestyle and income on medical students' career specialty choices: data from two US medical schools, 1998-2004. Acad Med 2005; 80: 809-14.

10. Avgerinos ED, Msaouel P, Koussidis GA, Keramaris NC, Bessas Y, Gourgoulianis K. Greek medical students' career choices indicate strong tendency towards specialization and training abroad. Health Policy 2006; 79: 101-6.

11. Borracci RA, Pittaluga RD, Manente D, Giorgi MA, Rubio M. Expectativas de los estudiantes de medicina de la Universidad de Buenos Aires sobre su práctica profesional. Medicina (B Aires) 2009; 69: 607-11. 